\title{
NEW FAST DETECTION METHOD OF FOREST FIRE MONITORING AND APPLICATION BASED ON FY-1D/MVISR DATA
}

Jianzhong Feng ${ }^{1,3, *}$, Huajun Tang ${ }^{1}$, Linyan Bai ${ }^{2}$, Qingbo Zhou ${ }^{1}$, Zhongxin Chen ${ }^{1}$

1 Institute of Agricultural Resources and Regional Planning, Chinese Academy of Agricultural Sciences, Beijing, China, 100081

2 Institute of Remote Sensing Applications, Chinese Academy of Science, Beijing, China, 100101

${ }^{3}$ Department of Surveying and Land Science, China University of Mining and Technology, Beijing, China, 100083

* Corresponding author, Address: Key Laboratory of Resources Remote Sensing \& Digital Agriculture, Institute of Agricultural Resources and Regional Planning, Chinese Academy of Agricultural Sciences, No. 12, South Zhongguancun Street, Beijing, 100083, China, Tel: +86-13522241414, Email: fengjzh4680@sina.com.cn

Abstract: In this paper, the authors proposed a new work and process flow algorithm about remote sensing image data to forest fire identification and monitoring, which was greatly different with the traditional approaches. Therefore, a more useful context method was used to detect forest fire spots, banes on statistic rationale, meanwhile the cloud-contaminated pixels were rejected (if any) and the misjudged fire spots excluded by examination of NDVIs before and after forest fires occurred; moreover, a simpler and more resultful approach was used to earth-locate forest fire spots, and a forest-fire sub-pixel area situation was taken into account so as to evaluate forest-fire area and enhance accuracy of evaluated forest fire areas. Subsequently, a FFDM prototype system was designed and developed and later tested using the typical FY-1D/MVISR data, performance and running efficiency of which were practically greater than the normal mainstream software systems (e.g. ENVI) in this domain.

Keywords: FY-1D/MVISR, Radiance, vegetation index, geo-referance, workflow

Feng, J., Tang, H., Bai, L., Zhou, Q. and Chen, Z., 2008, in IFIP International Federation for Information Processing, Volume 258; Computer and Computing Technologies in Agriculture, Vol. 1; Daoliang Li; (Boston: Springer), pp. 613-628. 


\section{INTRODUCTION}

Forest Fires are a prominent multi-scale phenomenon, which not only destroy natural vegetation, but also pose enormous danger to wildlife as well as to human life and property, so researchers, multi-level governments and others pay great attention to them. In view of preventing them from occurring in danger areas, there is one of crucial problems that is both smartly and in time to monitoring situation of and achieve environmental change of forest fires, but it is evident that the objectives couldn't be reached, if only using approaches and information of ground-based observation and monitoring, especially on large-scale forest fires. Relying on satellite remote sensing data, a wide-range and real-time monitoring is the most feasible and practical since it is essential for the grasp of fire occurrence situation and useful information that is half-automatically or automatically extracted from those data.

A surprising number of satellite systems are currently available, providing data and other capabilities that can be used for different aspects of forest fire monitoring since the late 1990s (Christopher et al., 2003). For instance, there are very common utilities of the NOAA/AVHRR (Advanced Very High Resolution Radiometer) and EOS (NASA Earth Observing System)/MODIS (Moderate Resolution Imaging Spectroradiometer) data in a large or global scale of satellite forest fire monitoring. The NOAA/AVHRR data are characterized with low cost, high temporal resolution (i.e., two daily passes per day over a given area for each operating satellite, thus two scenes obtained by each operational platform) and pantoscopic scan, as compared with the EOS/MODIS data that are greatly improved in spatial resolution, have more spectral channels (the total of 36 spectral channels), offer a larger dynamic range of radiance values, and enhance derived products and so on (though being somewhat lower in temporal resolution). The MVISR (Multichannel Visible and Infrared Scan Radiometer), onboard the present operating FY-1D satellite (which is a polar-orbiting meteorological satellite, developed and launched by China), is largely similar to the NOAA/AVHRR in aspects of functionalities and performances. It has 10 spectral channels, the corresponding ranges of which are same to the NOAA/AVHRRs', thereby the FY-1D/MVISR data show greatly potential for a large scale or global forest fire monitoring.

In the forest fire monitoring, active fire or hot spot detection is one of underlying tasks using satellite data. So far, methodologies of these detecting researches are abundantly achieved, based on satellite remote sensing systems and their obtained data, especially on the NOAA/AVHRR. Take for examples, Kalpoma Kazi A. et al. proposed a new algorithm of forest fire detection method with statistical analysis using NOAA/AVHRR images (Kalpoma et al., 2006); Galindo I. et al. carried through real-time 
NOAA/AVHRR forest fire detection in Mexico (Galindo et al., 2003); it was acquired to study a RS (using NOAA/AVHRR data)-and-GIS-integrated forest fire monitoring (Zhang, 2004). With respect to different satellite data, more effective and efficient algorithms of active fire or hot spot detection and other technologies, however, are still explored by researchers/users, in order to monitor forest fires and prevent their from emerging or reduce their losses in quite broad areas.

In this paper, the authors present a new work and process flow algorithm about remote sensing image data to forest fire identification and monitoring, which is different from traditional approaches. Useful and interesting information of forest fire spots is estimated and extracted from potential forest-fire pixels above all, which lies on corresponding thresholds determined with a statistic manner that validly eliminates subjective influences in a certain context and satisfies real requirements, meanwhile the cloud-contaminated pixels are rejected (if any) and the misjudged fire spots excluded by examination of Vegetation Index (VI) before and after forest fires occur; and forest fire spots are located and assigned exactly geocoordinates, using an interpolation algorithm of fast location of forest fire spots, rather than all unavailable and forest-fire-free spots; in addition, forest-fire sub-pixel area status is taken into account so as to evaluate forestfire area and enhance accuracy of evaluated forest fire areas. Subsequently, a FFDM prototype system is designed and developed and later tested using the typical FY-1D/MVISR remote sensing data involved in forest fires, while compared to normal mainstream software systems (e.g. ENVI \& ERDAS). In general, this prototype is to certain extent valuable and meaningful for FFDM in theoretic and applicable domains.

\section{METHODOLOGICAL MODES}

According to Wien's Displacement Law, a wavelength of thermal radiation with a peak is inversed to some absolute temperature of blackbody (Chen, 1985). Thus, there is a peak (about $9.7 \mu \mathrm{m}$ ) of thermal value while at the normal temperature (about 300k); meanwhile, related to the FY1D/MVISR data, there are more thermal radiant fluxes in $\mathrm{CH} 4$ $(10.3-11.3 \mu \mathrm{m})$ and $\mathrm{CH} 5 \quad(11.5-12.5 \mu \mathrm{m})$. As appears a brightness temperature of fire spot (a active fire or hot spot signature), thermal peak values transfers towards $\mathrm{CH} 3$ (e.g., a peak value about $5.8 \mu \mathrm{m}$ to the temperature $500 \mathrm{k}$ ), and there arise energies of observed thermal radiation, as well as are more in $\mathrm{CH} 3 \& 4$. Variation ratios of brightness temperatures, therefore, are normally evaluated and their trends depicted in $\mathrm{CH} 3 \& 4$, using the Planck function of blackbody radiation. It is obvious that there are 
distinguishing differences between in $\mathrm{CH} 3 \& 4$ (e.g., when temperatures of blackbody arising from $300 \mathrm{~K}$ to $500 \mathrm{~K}$ and even to the higher, a several hundredfold increase of variation ratios for the fore, but only over 10 times a increase for the latter) (Che et al., 1900). In FY-1D/MVISR image data, the pixel graylevel value of hot spot (including fire spot) is enormously different from those of its surrounding pixels in $\mathrm{CH} 3$, in contrast with the fact that there are only a few graylevel differences for the related pixels in $\mathrm{CH} 4$.

Forest fire detection procedures may be based on the fixed threshold value or contextual method, but there are lots of limits in the adoptability of the fore because of hugely relying on empirical analyses. The contextual method is devised to take into account the relationships between observational pixels and its neighboring pixels mainly based on spatial statistics, and then identify fire pixels from around candidate fire pixels, hence the contextual approach is often preferred to be selected (Kudon, 2005) and also adopted in this paper.

\subsection{Detection of forest fire spots}

In procedure of identifying forest fire spots, the Planck function of blackbody radiation is shown as a fundamental rationale, from which related others are derived. As the following below:

$$
T=h \cdot c /\left(k \cdot \lambda \cdot \log \left(2 \cdot h \cdot c^{2} /\left(I \cdot \lambda^{5}\right)+1\right)\right)
$$

where $T$ is brightness temperature $(K) ; h, k$ and $c$ are the Planck, Boltzmann and light-velocity constants respectively, which are $6.6256 \cdot 10^{-34}(\mathrm{~J} \cdot \mathrm{s}), \quad 1.3906 \cdot 10^{-23}\left(\mathrm{~J} \cdot \mathrm{K}^{-1}\right) \quad$ and $2.998 \cdot 10^{8} \quad(\mathrm{~m} \cdot \mathrm{s}) ; \quad \lambda$ radiance wavelength $(m)$ and $T I$ emissivity, whereas $I$ may be obtained from the following function (i.e., the radiometric calibration formula).

$$
I=n \cdot g+b
$$

where $n$ is pixel graylevel value; $g$ is the gained value and $b$ the bias value, which are the calibration coefficients $\left(\mathrm{W} / \mathrm{m}^{2} \cdot \mathrm{ster} \cdot \mu \mathrm{m}\right)$. Formula (1) and (2) hence are jointly evaluated so that the thermal radiances, obtained in $\mathrm{CH} 3 \& 4$, are transformed into the corresponding brightness temperatures.

Now it is a key task of detecting fire spots how to identify fire pixels, associated with the real fire spots, from potential fire pixels in satellite image, as below is followed (Kudoh, 2005; Giglio et al., 2003; Zhou et al., 2006): 


\section{- Definition of fire context window}

A fire pixel, selected by certain conditions (See the next sections), appears to be different enough from its background and meanwhile the brightness temperatures are employed, which are derived from the FY1D/MVISR in CH3\&4, respectively denoted by $T_{3}$ and $T_{4}$. Statistical information of each potential-fire pixel is calculated for a variable size context window around it, within which at least $25 \%$ of neighboring pixels are satisfied with forest fire-free pixels and considered as forest fire background, but not cloud-contaminated (If any, cloud pixels are rejected from the forest fire background because they are obscured. See identification of Cloud below).

\section{- Identification of Cloud}

Cloud identification is performed using a technique based on conditions: Reflectance $_{1}>22 \%$ and Reflectance $_{2}>23 \%$ that are respectively derived from the FY-1D/MVISR data in $\mathrm{CH} 1 \& 2$, and $T_{4}<273 \mathrm{~K}$. If the previous conditions are jointly be satisfied, then the pixels in the FY-1D/MVISR may be flagged as cloud (Zhang, 1999).

\section{- Identification of potential-fire pixels}

If $T_{3}>320 \mathrm{~K}$ and $\square T_{34}>20 \mathrm{~K}$ in daytime, or $T_{3}>315 \mathrm{~K}$ and $\square T_{34}>10 \mathrm{~K}$ in nighttime, where $\square T_{34}=T_{3}-T_{4}$, then a pixel is identified as a potential fire pixel.

\section{- $\quad$ Tentative identification of fire pixels}

If the potential-fire pixels are identified as active fire (including hot spots), the following conditions below are founded on:

$$
\begin{aligned}
& T_{3}>\overline{T_{3}}+4 \delta T_{3}, \text { where, if } \overline{T_{3}}<2 \mathrm{~K} \text {, then } \overline{T_{3}}=2 \mathrm{~K} \\
& \square T_{34}>\overline{\square T_{34}}+4 \delta T_{34} \\
& T_{4}>320 \mathrm{~K} \text { in daytime (or } T_{4}>315 \mathrm{~K} \text { in nighttime) } \\
& \square T_{34}>20 \mathrm{~K} \text { in daytime (or } \square T_{34}>10 \mathrm{~K} \text { in nighttime) } \\
& T_{3}>360 \mathrm{~K} \text { in daytime (or } T_{3}>330 \mathrm{~K} \text { in nighttime) }
\end{aligned}
$$

where $\bar{T}_{3}$ and $\delta T_{3}$ are the respective mean and mean square deviation of $T_{3}$ for the valid potential-fire pixels in the background; $\overline{\square T_{34}}$ and $\delta T_{34}$, the respective mean and mean square deviation of $\square T_{34}$ for the valid potentialfire pixels in the background.

In the subsequent sections, the valid potential-fire pixels are tentatively identified as fire pixels using the below conditions, as are described later on: 
If $\{((3)$ or $(5))$ and $((4)$ or $(6))$ or $(7)\}$ is true, where, if $\delta T_{3}$ or $\square T_{34}<2 K$, they are assigned with $2 K$; or else, they are maintained and invariable,

Then the above valid potential-fire pixels are figured as fire pixels (fire spots).

\subsection{Location of forest fire spots}

To date, fire pixels have been detected in the satellite images. The next task is that identified fire spots are, accurately and quickly, located and assigned with corresponding geo-coordinates, hereby an interpolation algorithm of fast location of forest fire spots, based on the FY-1D/MVISR data, is discussed so as to better serve the direct or derived productions to related departments (e.g. helping for efficient mapping of forest fires).

The FY-1D/MVISR is characterized that there are 2048 counts (i.e. samples) along each scan, each of which is 10-bit binary values (in each spectral channel) and archived into the format of 2 bytes (one per 8-bit byte) in storing system. Based on the 1a.5 file format, earth-location of data is possible using benchmark data provided with each archived sector. The benchmark data consist of line and sample number coordinates of data samples falling on certain span increment of latitude and longitude; furthermore there are latitudes and longitudes of 51 benchmark data points along each scan in the file (bytes 151 to 354), each pair of which are archived into 4 bytes.

The authors thus employ the nearest-neighbor infill pixel interpolation algorithm and WGS-84 Coordinate System to geo-rectify FY-1D/MVISR image datum, and obtain an orthoimage and associated geo-coordinate information file (storing the first langitude and latitude and adjacent pixels' difference values respectively between longitudes and latitudes, which are mapped into the associated orthoimage), in order to obtain the longitude and latitude values of center of an arbitrary pixel in the orthoimage and then earth-locate the corresponding forest fire spots. There is a basic algorithmic despcription of earth location in an associated geo-coordinate information file, as is shown below:

FUNCTION fire_spot_location (fnm, $i, j)$

$\{/ *$ fnm is an orthoimage file; and $i, j$ are the longitude and latitude values of center of an aimed pixel $* /$

map_info $=$ get_map_info $(f i d=f n m)$;

lon_ini = map_info.mc[2]; /*Obtaining the longitude value of center of the first pixel in the orthoimage*/

lat_ini=map_info.mc[3]; /*Obtaining the latitude value of center of the first pixel in the orthoimage*/

lon_interval $=$ map_info.ps[0]; $/ *$ Obtaining difference values of the most-adjacent pixels between longitudes*/ 
lat_interval $=$ map_info.ps[1]; /*Obtaining difference values of the most-adjacent pixels between latitudes $* /$

lon_j $=$ lon_ini $+(j-1) *$ lon_interval; $/ *$ Calculating the longitude value of center of the pixel $(i, j) * /$

lat_i $=$ lng_ini $+(i-1) *$ lng_interval; $/ *$ Calculating the latitude value of center of the pixel $(i, j) * /$

.....

\}

\subsection{Examination of Vegetation Index (VI)}

When the actual forest fire detection and monitoring are carried out, the observed values just through an infrared channel are inversed to obtain the brightness temperature and determine whether the forest fires occur, but owing to the complexity of land surface, there are often lots of misjudgments (e.g., a high temperature desert area would be misjudged into a forest fire area; and smoking chimneys or other special high heat sources into forest fire spots). It is a good way that the vegetation index (VI) is used to solve the problem. Obviously, the VIs are some significant digital values and indicate vegetation growth status, biomass and so on (Jiang et al., 2001). According to the Satellite image data in the recent period of time before the forest fires occurred, the VIs whose earth-locations are corresponded with the identified potential forest-fire pixels, therefore, are calculated to define identification credibility of forest fire spots and determine whether the potential forest fire spots are real or not: the VIs are higher, and the real probabilities of potential forest fire spots are bigger; furthermore, to evaluate areas of or assess other disaster losses of forest fires and so forth in terms of the VIs changes before and after the forest fires take place.

Up to now, reseachers have studied and developed dozens of different models of the VIs that are widely applied (Xu, 1991). In this paper, a model of Normalized Difference vegetation index $(N D V I)$ is expressed below:

$$
N D V I=\frac{\rho_{N I R}-\rho_{R}}{\rho_{N I R}+\rho_{R}}
$$

Where $\rho_{\text {NIR }}$ and $\rho_{R}$ are the reflectances of land surface in the nearinfrared and red bands, respectively.

As it is charactered with the design of and data acquisition mode of channels of FY-1D/MVISR, the correponding FY-1D/MVISR data in CH1 $(0.58-0.68 \mu \mathrm{m})$ and $\mathrm{CH} 10(0.90-0.96 \mu \mathrm{m})$ are preprocessed by a series of ways such as radiometric calibration and solar elevation correction, and then the $\rho_{\text {NIR }}$ and $\rho_{R}$ are obtained to calculate corresponding NDVI values and detect forest fire spots or assess forest fire situation and etc. 


\subsection{Evaluation of forest fire areas}

Evaluation of forest fire areas is a very important task of forest fire losses assessment and one intention of detecting and monitoring forest fire. In some cases, a real fire spot is so small that its area is less than a resolution of one pixel, and therefore, a sub-pixel scale should be taken into account necessarily to evaluation of forest fire areas.

Assuming a rate $P$ is a sub-pixel forest fire area over the area of a pixel (i.e. a field of view), there exists $1-P$ that is a sub-pixel forest-fire-free area within a field of view, so the radiant flux density equation below is expressed, saturated in $\mathrm{CH} 3$ of the FY-1D/MVISR:

$$
E\left(\lambda, T_{3}\right)=p \cdot E\left(\lambda, T_{f}\right)+(1-p) \cdot E\left(\lambda, T_{b}\right)
$$

where $E$ is a Planck deformation function; $\lambda$ a detection wavelength in $\mathrm{CH} 3 ; T_{3}$ a pixel land surface temperature in $\mathrm{CH} 3 ; T_{f}$ a forest fire temperature for a sub-pixel in $\mathrm{CH} 3 ; T_{b}$ a fire-free area temperature for a pixel (i.e. a background temperature). In the view of mathematic theory, a binary equation group about $T_{f}$ and $T_{b}$ may be founded and solved so as to obtain the $T_{f}$ and $T_{b}$ values, which is based on the Planck function $E(\square)$; nevertheless, given that $E(\square)$ is non-linear, it is very difficult and even impossible to practically solve the equation group and gain the above rate $P$. In practice, a logical value $T_{f}$ is often experientially set on the basis of the actual instances, or, relying on the pertinent relationship, a certain fitting function upon $T_{f}$ is constructed to obtain the needed $T_{f}$ a value $T_{b}$ is derived from the mean temperature for the 8 immediate neighboring pixels around a forest fire pixel (Xu, 1991); and then the rate $P$ is obtained, using Equation (9). Necessarily noting, given that $T_{3}$ is obviously more than $T_{b}$ and often $T_{f}$ over $500 \mathrm{~K}$, and a positive relationship for Variable $E$ with Temperature $T$ in the previous $E(\square)$, a obtained rate $P$ should fall between 0 and 1; otherwise it is invalid, so causes have to be discovered and the values be revised to achieve a sound rate $P$ (Dong et al., 1999). Now a sub-pixel forest fire area can be evaluated by $P$ multiplied by a corresponding pixel forest fire area.

\section{PROCESS AND WORKFLOW}

According to the previous rationale and methods about forest fire detection, the process and workflow of forest fire detection and monitoring are designed, as illustrated in Figure 1. 


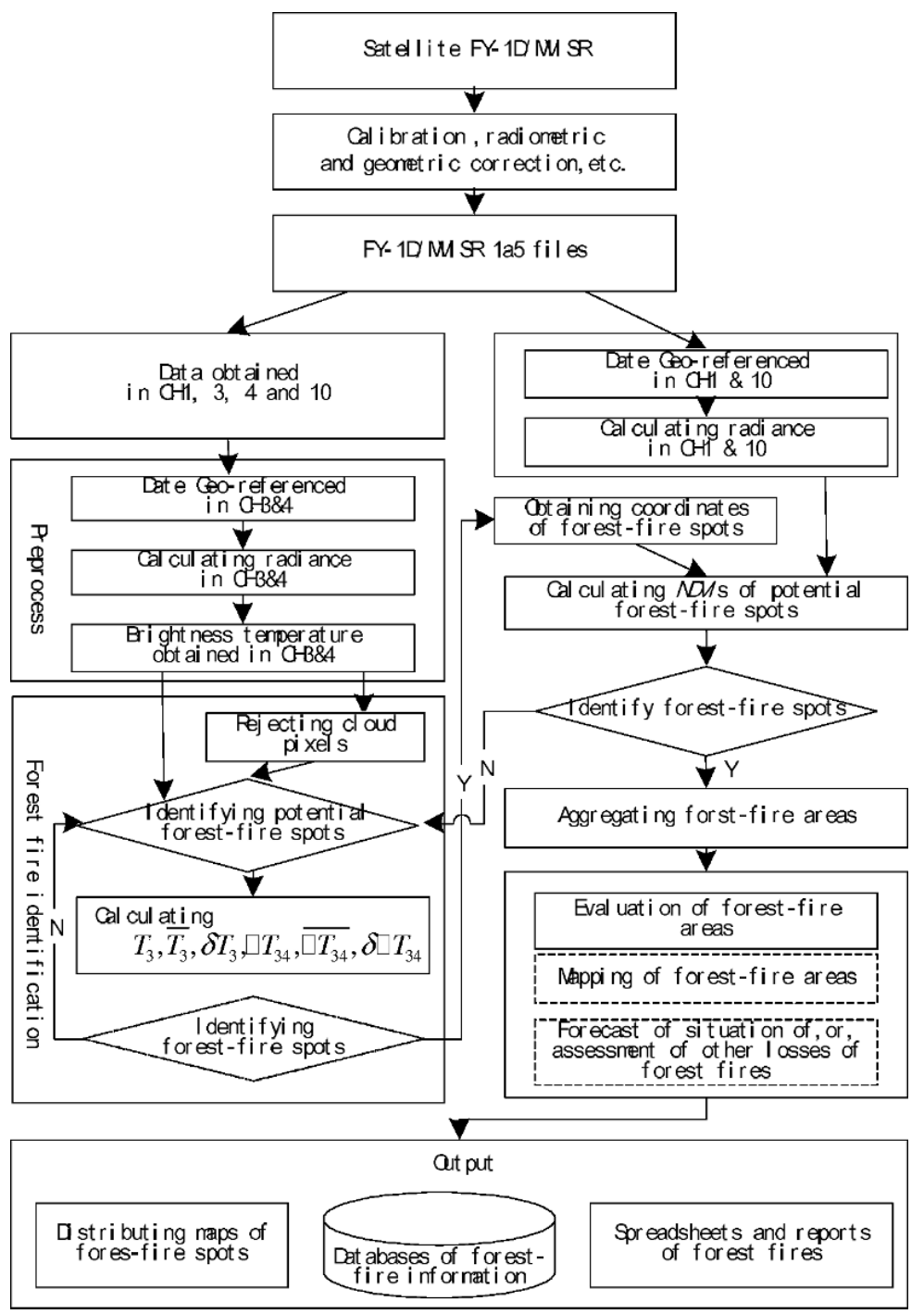

Figure 1. The process and workflow of forest fire detection

After the given raw FY-1D/MVISR data are preprocessed by a series of essential techniques such as calibration, radiometric and geometric corrections, the corresponding data products (i.e. called the $F Y-1 C / M V I S R$ product data) may be obtained and ultimately act on the next test of forest fire detection and monitoring. It obviously shows that the forest fire detection approach is a pivotal and complex task that is determined by a series of factors and conditions. The context method, based on statistics, is used to obtain the decision thresholds and identify the potential pixels in the background, in order that the great deviations or errors, resulted from the 
subjective factors or decisions of forest fire detection, are reduced and even avoided; meanwhile, the cloud-contaminated pixels are rejected, and the NDVIs used to be compared before and after forest fires occure, so as to usefully obtain the real forest fire spots in the end (see Section $2.1 \& 2.3$ ). Parallelly, earth location of the forest fire spots is carried out to obtain their geo-coordinates. The attitude and longitude coordinates of forest fire spots are gained in accordance with the spatial distributing feature of the line and sample number coordinates of benchmark data points, using some listed and suitable algorithm and complying with the neighboring-benchmark-point rule (see Section Location of forest fire spots).

So far, there are subsequent tasks such as aggregation and mapping of forest fire spots, and evaluation of forest fire areas (see Section Examination of VI); moreover, it is necessary to take notice of sub-pixel status for forest fires, for example a pixel area is divided to aggregate the sub-pixel forest fire spots in certain rules (Xu et al., 2001). Lastly, the upper obtained data products are output and archived with the map and documentation files, and spreadsheets, etc, or directly stored into or used to update the forest-fire informational databases, including plenty of the related basic information such as the geo-coordinates of forest fire spots, time and areas of forest fires, and useful parameters (e.g. $T_{3}$ ).

\section{DESIGN AND IMPLEMENTATION}

As is mentioned previously, a prototype system about forest fire detection and monitoring (FFDM) is designed and developed, which correspond to the user-driven processing and is able to run in a wide hardware and software environment (e.g. workstation, PC computer, and Windows, Linux). It can easily and effectively enhance the capabilities of FFDM and provide a helpful tool for the associated management and making-decision departments to differentiate forest fire areas, further prevent forest fires from occurring, even plan the burned scars and proceed to recover ecological environment and so on. In general, there are a few primary functionalities in the overall system, containing information extraction of forest fires, identification and earth-location of forest fire spots, and visual display of related information and processing results, etc.

The architecture of the FFDM prototype mainly consists of a series of functional subsystems, as presents in Figure 2: (1) the information extraction subsystem presents to extract information/data as data sources of next forest fire detection from the FY-1D/MVISR (e.g., the spectral information in $\mathrm{CH} 3 \& 4$, the line and sample number coordinates of benchmark data points, and image sampling time); (2) the threshold determination subsystem is 


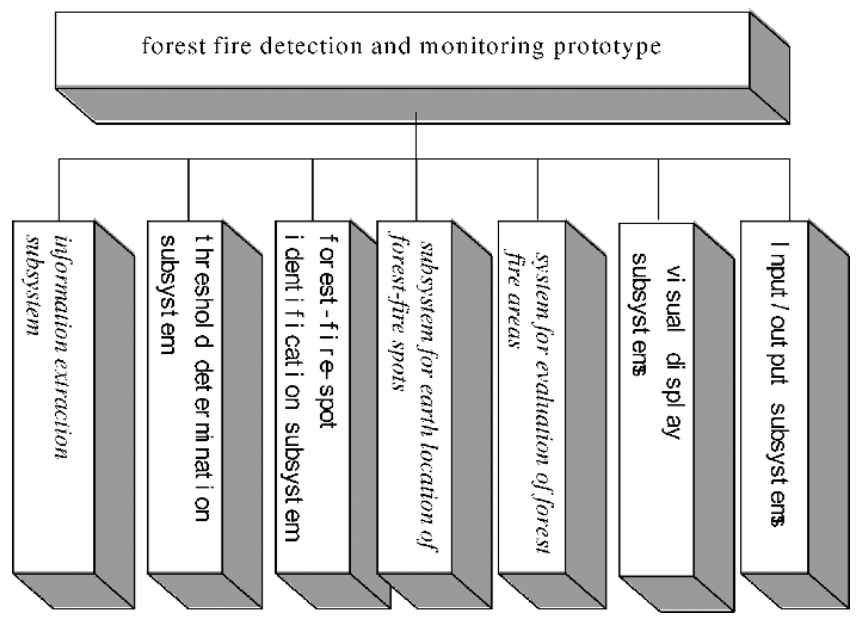

Figure 2. A framework of FFDM system

responsible to the valid thresholds that are obtained in statistic manner (see Section 2.1), using the brightness temperatures transformed from the previous acquired spectral data; (3) the forest-fire-spot identification subsystem is responsible to identify the forest-fire spots from the potential forest fires in the background and reject the cloud-contaminated areas (if any) and exclude the misjudged fire spots by the NDVIs; (4) the subsystem for earth location of forest-fire spots assigns the identified fire pixels with the corresponding geo-coordinates (of course, as well as is available for the forest-fire-free common pixels); (5) the system for evaluation of forest fire areas, including the referred sub-pixel areas; (6) the Input/output and visual display subsystems present input/output functions with user-friendly man-andcomputer interactive mode, especially upon visual display. In addition, the databases subsystem of FFDM is a basic and pivotal component in the overall FFDM system. It can make the related forest-fire information/data very availably stored, accessed managed, and analyzed, etc, thus abilities and levels of analyzing and processing problems in the FFDM system are improved in effect and further all performances of the FFDM system are heightened as well. For instance, Figure 3 shows the structures and relationships of partial spatial database tables about forest fire detection in this system.

Furthermore, parts of the implemented results as to the FFDM system are demonstrated (see Figure 4 and 5). In this system, the data structures are constructed in accordance to the stored scheme of the FY-1D/MVISR 1A.5 files so that the associated available information is extracted. Fig. 5 shows the file-head information extracted from some 1A.5 file, including the ID and orbital parameters and of the satellite, time of data acquirement, and "good" 


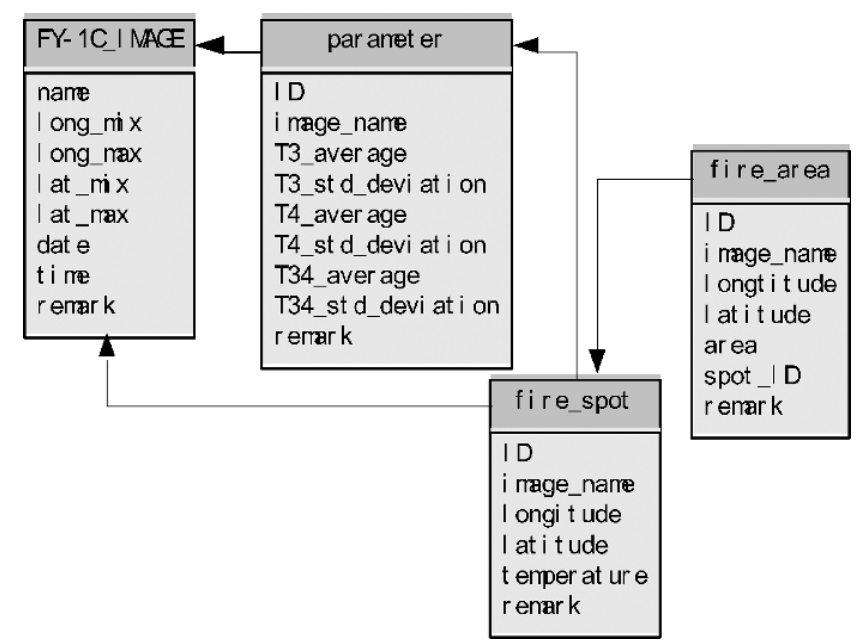

Figure 3. Structures and relationships of partial spatial database tables about forest fire detection in the FFDM system*

*(a) FY-1D_IMAGE table stores the information FY-1D/MVISR Image data (including the related basic information); (b) parameter table stores the corresponding calculated parameters of forest fire detection; (c) fire_spot table records the detected spot information; (d) fire_area table stores the information of forest-fire-spot-aggregated areas (e.g. the remark regarding fire-area features, and evaluated area, etc).

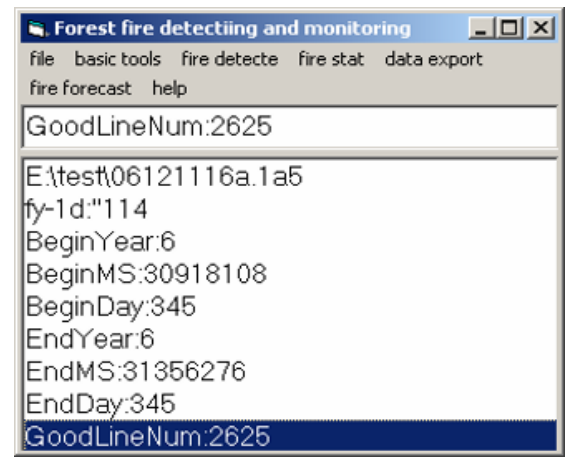

Figure 4. Interface of file-head information extraction for FY-1D/MVISR data

lines (i.e. valid lines) number in the file, the calibration coefficients and standard deviations of data in Channels 1 to 10, and geo-coordinates of 4 edge corners of cover areas, etc. Additionally, the FFDM system presents two patterns of the forest fire automatic and manual detection: if the former selected, an user can obtain the related data in some channels, or, he can manually obtained the needed spectral data (including other basic information) in chose channels (see Figure 5), as well as the obtained related spectral data are stored in the format of *.txt files that contain the spectral bare data file, band parameter file, benchmark-data-point geo-coordinates file and log file, etc, whereas these are only illuminated to data/data-files, although this system are characterized in some other ways. 


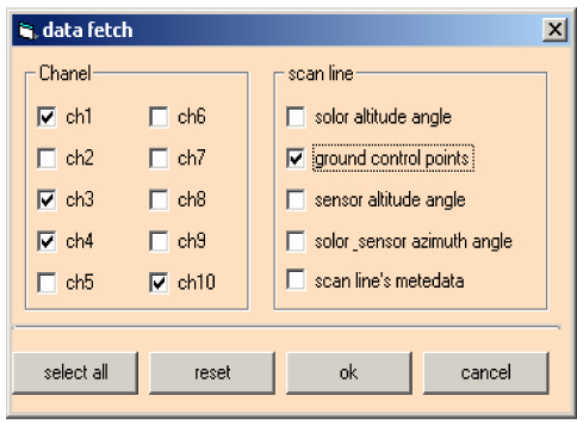

Figure 5. Interface of spectral-band information extraction

\section{ANALYSIS AND DISCUSSION}

Given the FY-1D/MVISR data of 5 scenes (implicating forest fire information) in China, downloaded from the site (http://www.ers.ac.cn), this FFDM system hence was tested and investigated in the application. By testing, the system demonstrated its high performance and great processing competences and satisfied the valid accuracy and precision requirements of FFDM application with the user-friendly operation interfaces and tools. It was particularly notable that the FFDM prototype offered two operational approaches mentioned above, i.e. the automatic and manual processes: choosing the fore, the user only clicked "File" to "Open", and immediately a window pop up, and the user selected the needed FY-1D/MVISR image file, where after the functions of system were completed such as identification and earth location of forest fire spots, and fire area division, and producing the reports of fire situation, etc, which needed not to be operated by the user, but this system could automatically proceed to operate; if the latter selected, it is necessary to manually complete the related operation. Additionally, the results of processing data were stored into the forest-fire informational databases so as to be retrieved, queried and further processed, and concurrently the associated forest-fire map (see Figure 6, 7 and 8), handingin spreadsheet and report products and so on.

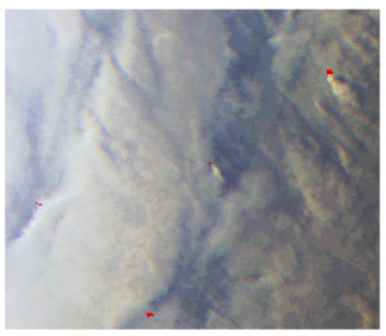

Figure 6. A distribution map of potential forest-fire spots

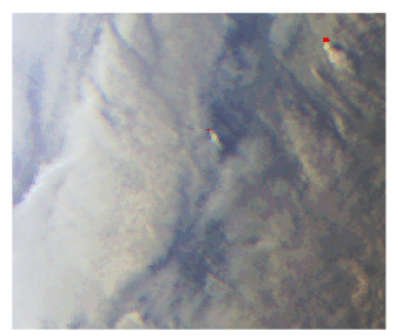

Figure 7. A distribution map of actual forest-fire spots

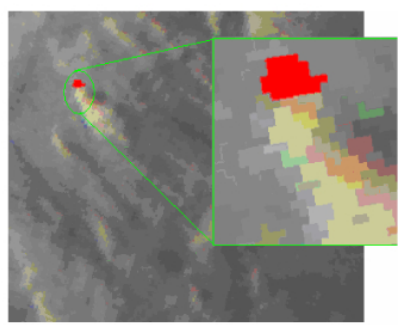

Figure 8. Status of a forest fire area 
Because the new workflow scheme and fast earth location algorithm of forest fire detection were employed in the FFDM system, calculation contents of forest fire detection were much more reduced, and cost time of processing image data greatly shortened, and performance of the system enormously improved, for example in Table 1 there lists the relevant information of selected image data and process results (i.e., consuming time of data process ).

Table 1. Executing efficient status of forest fire detection*

\begin{tabular}{rrcc}
\hline Name of data files & Samples*lines & Data size (Mbety) & Processing time (Second) \\
\hline 07022607.1a5 & $2048 * 3665$ & 155 & 350 \\
$07031005.1 \mathrm{a} 5$ & $2048 * 3288$ & 138 & 309 \\
$07030919.1 \mathrm{a} 5$ & $2048 * 2304$ & 97.5 & 225 \\
$07050406.1 \mathrm{a} 5$ & $2048 * 3584$ & 151 & 320 \\
$07051606.1 \mathrm{a} 5$ & $2048 * 3606$ & 152 & 324 \\
\hline
\end{tabular}

*Main parameters of configuration about the computer executing the programs: Intel ${ }^{\circledR} \mathrm{P} 4$ 2.4GHz, 512 RAM; the FY-1D/MVISR 1A.5 data source from the site of Remote Sensing of Evironment in China, http://www.ers.ac.cn

At present, there are no special subsystems to process the FY-1D/MVISR data in many mainstream software systems of remote sensing image process (e.g. ENVI \& Erdase); therefore, if using them, it is difficult and complex to directly process the remote sensing data in some application ways. Take an example, in some forest fire detection and monitoring there exist complicated steps and users rely on manual operations step-by-step to fulfill related tasks (e.g. many of parameters need to be configured in detail, merely related to opening a FY-1D/MVISR data file, using the ENVI software system; and geometric correction, forest fire identification and so forth are further more perplexing), so efficiency of forest fire detection and monitoring is very lower. In the sake to overcome the mentioned difficulties and faults, in this research a new workflow algorithm of forest fire detection and fast forest-fire earth location scheme were designed and developed. As a result, the FFMD system demonstrated the high task efficiency and nice performance, and was useful and worth of academic and applicable domains.

\section{CONCLUSIONS}

In this research, the authors employed a new workflow algorithm of forest fire detection using the remote sensing image data, which is greatly different with the traditional approaches. For the new algorithm, the useful 
context method was used to detect forest fire spots that were identified from potential forest-fire pixels, which relied on corresponding thresholds determined with a statistic manner that validly eliminated subjective influences in a certain context and satisfied real requirements, meanwhile the cloud-contaminated pixels are rejected (if any) and the misjudged fire spots excluded by examination of NDVIs before and after forest fires occur; moreover, earth location of forest fire spots was simpler and more resultful because it referred to the available forest fire spots/areas, in which users were interested rather than in unavailable spots/areas, so calculation contents of forest fire detection were more reduced and process running time of system also greatly lowered; additionally, a forest-fire sub-pixel area situation was taken into account so as to evaluate forest-fire area and enhance accuracy of evaluated forest fire areas.

Consequently, a FFDM prototype system was designed and developed and later tested using the typical FY-1D/MVISR remote sensing data involved in forest fires. Practically, performance and running efficiency of the prototype are greater than the normal mainstream software systems (e.g. ENVI \& ERDAS) with respect to process and operation of the FY-1D/MVISR data; therefore, it was very beneficial and worth of related departments dealing with fire detection, control, prevention and further burned scars plan and ecological recovery, etc. In short, the system are greatly propitious to forest fire detection, monitoring, management and so the like. Lots of researches, however, need still further to be carried out both theoretically and methodologically in order to be applied to wider application domains.

\section{ACKNOWLEDGEMENTS}

This study is supported by the China National High Technology Research and Development Program (863 Program) Project (No. 2006AA12Z103) and China National Key Technologies R\&D Program Project (No. 2006BAD10A06).

\section{REFERENCES}

Chen H. (1985). Thermal infrared physics. National Defence Industry Press, Beijing

Che N. Z., Yan D. Y. (1900). Radiometry and photometry. Beijing institute of technology Press, Beijing

Christopher O. Justice, Smith R., Gill A. M., Csiszard I. (2003). A review of current spacebased fire monitoring in Australia and the GOFC/GOLD program for international coordination. International Journal of Wildland Fire, 12, 247-258 
Dong C. H., Zhang G. C. (1999). Xing F. Y. et al., Tool manual of meteorological satellites, China Meteopoiogical Press

Galindo I., Lopez-Perez P., Evangelista-Salazar M. (2003) Real-time AVHRR forest fire detection in Mexico (1998-2000). International Journal of Remote Sensing, 24(1), 9-22

Jiang D., Wang A. B., Yang X. H., Lu H. H. (2001). Ecological Connotation and Application of the Vegetation Index-Surface Temperature Feature Space. Progress in geography, 20(2), $146-152$

Kudoh J. I. (2005). Forest fire analysis for several years in Russia by using NOAA satellite http://www2.cr.chiba-u.jp/symp2005/documents/Oralsession/Session3_Dec14/S32JunichiKudoh_paper.pdf

Kalpoma K. A., Kudoh J. I. (2006). A new algorithm for forest fire detection method with statistical analysis using NOAA AVHRR images, International Journal of Remote Sensing, 27(18), 3867-3880

Giglio L., Descloitres J., Christopher O. Justice, Yoram J. Kaufman (2003). An Enhanced Contextual Fire Detection Algorithm for MODIS, Remote Sensing of Environment, 87, 273-282

Xu X. R. (1991). Processing of the Remote Sensing of Evironment Monitoring and Crop Yield Estimation, Peking University Press

XU X. R., Chen L. F., Zhuang J. L. (2001). Genetic inverse algorithm for retrieval of component temperature of mixed pixel by multi-angle thermal infrared remote sensing data. Science in China (Series D), 44(4), 363-372

Zhang C. G. (2004). Study on RS-GIS-based Forest Fire Monitoring in Fujian Provinc, Journal of Fujian College of Forestry, 24(1), 32-35

Zhang C. G. (1999). A Preliminary Study of Cloud Detection in remote sensing applications of Polar-orbiting Meteorological Satellites, Journal of Fujian Meteorology, 2, 3-5

Zhou X. C., Wang X. Q. (2006). Validate and Improvement on Arithmetic of Identifying Forest Fire Based on EOS-MODIS Data, Remote sensing technology and application, 21(3), 206-211 